\title{
Under Pressure DFT Investigations on Optical and Electronic Properties of $\mathrm{PbZrO}_{3}$
}

\author{
G. NAZIR ${ }^{a, b}, \mathrm{SAAD}_{\mathrm{TARIQ}}^{a, c, *}, \mathrm{~A} \cdot \mathrm{AFAQ}^{a}, \mathrm{Q} \cdot \mathrm{MAHMOOD}^{d}, \mathrm{~S} \cdot \mathrm{SAAD}^{c}, \mathrm{~A} \cdot \mathrm{MAHMOOD}^{d}$ \\ AND SAMAR TARIQ ${ }^{a}$ \\ ${ }^{a}$ Centre of Excellence in Solid State Physics, University of the Punjab, 54000, Lahore, Pakistan
}

${ }^{b}$ Department of Physics and Astronomy and Graphene Research Institute, Sejong University, Seoul 05006, Korea

${ }^{c}$ Centre for High Energy Physics, University of the Punjab, 54000, Lahore, Pakistan

${ }^{d}$ Institute of Physics, Centre for Advanced Studies in Physics, GC University, 54000, Lahore, Pakistan

${ }^{e}$ College of Engineering, Chemical Engineering Department, King Saud University, Riyadh, Saudi Arabia

(Received April 04, 2016; in final form November 28, 2017)

In this article, density functional theory has been used to investigate the structural and optoelectronic properties of $\mathrm{PbZrO}_{3}$ (PZO) under pressure from 0 to $350 \mathrm{GPa}$. In order to achieve ground state structural stability, generalized gradient approximations has been utilized. By studying electronic properties, indirect band-gap nature of PZO appears to change at $15 \mathrm{GPa}$ to direct band-gap. Optical analysis include under pressure responses of real and imaginary parts of dielectric function, optical conductivity, optical absorption coefficient, energy loss function, refractive index, reflectivity and extinction coefficient. Most of the results have been found to be consistent with literature. Study reveals that static dielectric constant and band-gap are in accordance with the Penn model which validates our computed results. Moreover, static dielectric constant and static refractive index directly increases with pressure. Material preserves its positive value of refractive index at all pressures and therefore, it is not a negative index metamaterial. Plasma frequency increases directly with pressure that destabilize the under study material. Our results could be very useful for developing novel optoelectronic devices based on PZO suitable to work under extreme conditions.

DOI: 10.12693/APhysPolA.133.105

PACS/topics: density functional theory, optical properties, electronic structure, high pressure

\section{Introduction}

Cubic perovskites exhibit remarkable electrical and optical properties along with immense variety of industrial applications. Lead zirconate, $\mathrm{PbZrO}_{3}$, is one of the distinguished representative of the family of perovskites. Extensive use of PZO in optoelectronic devices has made it applicable in infrared sensors, optoelectric modulators, memory storage devices, capacitors for high-frequency microwave devices, infrared detectors and numerous electromechanical systems [1-4]. Apart from magnificent electronic applications, PZO shows interesting optical properties, such as large values of refractive index, optical band-gaps and high transparency at infrared wavelengths. Such interesting features make the material a promising candidate for practical applications in various fields of integrated optics as sensors, optical shutters, optical waveguides, color filters, and ultrafast optical switches [5-9].

In literature, structure of PZO has been experimentally studied from X-ray and neutron diffraction techniques [10-18] while theoretical reports are based on density functional theory (DFT) [19, 20]. PZO has shown three stable phases: (antiferroelectric) orthorhombic phase up to $230^{\circ} \mathrm{C}$, (ferroelectric) rhombohedral

*corresponding author; e-mail: saadigi@hotmail.com phase ranging $230-233^{\circ} \mathrm{C}$ and a (paraelectric) cubic phase at above $233^{\circ} \mathrm{C}$. The dielectric behavior of PZO has been reported half a century ago [21, 22]. However, the crystal structure of the material is still under extensive study $[18,23]$. Density-functional calculations based on first principles have proved to be quite accurate in predicting ground state structural parameters of cubic perovskite oxides [24].

On theoretical grounds, number of researchers has reported structural and optoelectronic characteristics of cubic PZO by using DFT. Wang et al. [25] studied cubic PZO along (001) surface. Johannes and Singh [26] investigated the stability of cubic PZO using DFT calculations and found it suitable for electronic devices. Furthermore, Ghosez et al. [27] performed the phonon dispersion calculations of cubic PZO. Singh [28] has also investigated structural and electronic properties of cubic PZO by using local density approximations (LDA).

However, in literature, only few experimental data related to optoelectronic properties of PZO under high pressure have been reported. This article reports in detail under pressure study of PZO due to its technological and scientific importance. The details of sections are as follows. In Sect. 2, computational method explains theoretical formalism performed in this study. In Sect. 3, results and discussions include details of structural, electronic and optical properties in subsections of Sect. 3 as 3.1, 3.2, and 3.3, respectively. Finally, Sect. 4 concludes the paper. 


\section{Computational details}

Due to significant experimental applications of PZO and its existence in the stable cubic phase, we conducted theoretical study on structural and optoelectronic properties in order to explore the effects of PZO under extreme pressure. To achieve this, highly accurate full potential linearized augmented plane wave (FP-LAPW) [29] method with in the generalized gradient approximation (GGA) [30] embodied within WIEN2k code [31] has been used. In this well-known FP-LAPW method, the Slater theory of muffin-tin radii has been used in order to divide this space into two regions. Close to the atoms, all interesting quantities are expanded in terms of spherical harmonics and as expansion of plane waves with wave vector having cut-off value of $K_{\max }$ in the interstitial region. The earlier kind of expansion is started within a well-known muffin-tin sphere of radius $R_{\mathrm{MT}}$ around every nucleus. The choice of sphere radii predicts the rate of convergence of these expansions, but this affects only the speed of the calculation. Data calculated is for ideal bulk crystal of PZO with no defects. We choose $R_{\mathrm{MT}} K_{\max }=7$ as convergence parameters for the matrix element that represent number of fundamental functions, in which $K_{\max }$ is associated with plane wave cut-off in momentum space ( $k$-space) and $R_{\mathrm{MT}}$ represent the smallest radius of muffin-tin cube among all the atomic sphere radii.

Optical characteristics for PZO has been studied by dielectric function consisting of real and imaginary dielectric parameters. Following equations formulated by Ehrenreich and Cohen give brief description about the real and imaginary fragments of dielectric function $[32,33]$ :

$$
\varepsilon_{2}(\omega)=\frac{e^{2} h^{\prime}}{\pi m^{2} \omega^{2}} \sum_{v, c} \int_{\mathrm{BZ}}^{\infty}\left|M_{c v}(k)\right|^{2} \delta\left(\omega_{c v}(k)-\omega\right) \mathrm{d}^{3} k,(1)
$$

where the volume integral is limited to first Brillouin zone and dipole matrix element gives the information about direct transition between conduction band (CB) and valence band (VB) states. The relation gives the account of the excitation energy used during the transition between conduction and valence band states, $e$ represents polarization vector due to the electric field and gives detail about periodic portion of the Bloch wave function in conduction band associated with wave vector $k$. Furthermore, real part of dielectric function can be obtained from imaginary part of the Kramers-Kronig relation given by

$$
\varepsilon_{1}(\omega)=1+\frac{2}{\pi} P \int_{0}^{\infty} \frac{\omega^{\prime} \varepsilon_{2}\left(\omega^{\prime}\right)}{\omega^{\prime 2}-\omega^{2}} \mathrm{~d} \omega^{\prime}
$$

$\mathrm{P}$ denotes principal magnitude of integral. Refractive index is formulated as

$$
n(\omega)=\left(\frac{\varepsilon_{1}(\omega)}{2}+\frac{\sqrt{\varepsilon_{1}^{2}(\omega)+\varepsilon_{2}^{2}(\omega)}}{2}\right)^{1 / 2},
$$

where static refractive index is given as

$$
n(0)=\sqrt{\varepsilon(0)} .
$$

Similarly extinction coefficient is denoted by $k(\omega)$ and is calculated as

$$
k(\omega)=\left(\frac{-\varepsilon_{1}(\omega)}{2}+\frac{\sqrt{\varepsilon_{1}^{2}(\omega)+\varepsilon_{2}^{2}(\omega)}}{2}\right)^{1 / 2} .
$$

General formula of reflectivity to find the reflection from surface of any material is given as

$$
R=\frac{(n-1)^{2}+k^{2}}{(n+1)^{2}+k^{2}} .
$$

In the same way, energy loss function $L(\omega)$ [34], absorption coefficient $\alpha(\omega)$ and optical conductivity $\sigma(\omega)$ has been determined by using the following relation:

$$
\begin{aligned}
L(\omega) & =\operatorname{Im}(-1 / \tilde{\varepsilon}(\omega), \\
\alpha(\omega) & =4 \pi k(\omega) / \lambda, \\
\sigma(\omega) & =\left(2 W_{c v} h^{\prime} \omega\right) / E_{0},
\end{aligned}
$$

where $W_{c v}$ in the above relation represent transition probability per unit time.

\section{Results and discussions}

PZO belongs to a family of perovskite compounds [3537]. A cubic PZO structure $\mathrm{ABO}_{3}$ type has the space group $221 P m 3 m$ with the Wyckoff positions: $\mathrm{Pb}$ $(0,0,0), \mathrm{Zr} \overline{(0.5,0.5,0.5)}$ and $\mathrm{O}(0,0.5,0.5),(0.5,0.5,0)$ and $(0.5,0,0.5)$ as illustrated in Fig 1 . The relative response of PZO as a function high pressure has been demonstrated in terms of structural [38] and dielectric attributes [39]. In this section, we shall discuss in detail on structural, optical, and electronic properties of PZO. (a)

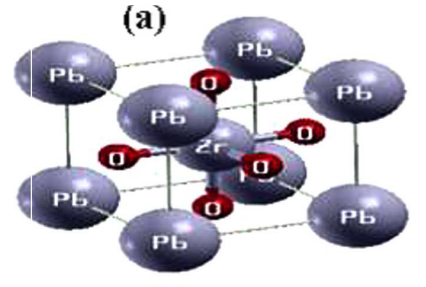

(b)

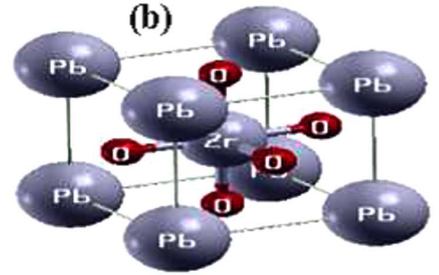

Fig. 1. Cubic PZO (a) ambient pressure, (b) 15 GPa.

\subsection{Structural properties}

In studying structural properties, lattice constant, bulk moduli, ground state energy-volume and derivative of bulk modulus have been calculated under pressure between 0 and $15 \mathrm{GPa}$ for PZO using FP-LAPW method within GGA. The calculated lattice constant for cubic $\mathrm{PZO}$ at ambient pressure equal to $4.19 \AA$ agrees well with experimental value of $4.1614 \AA$ [18], see Table I. Muffintin radius $R_{\mathrm{MT}}$ values of $2.5,1.96$, and 1.78 in atomic units (a.u.) have been used for $\mathrm{Pb}, \mathrm{Zr}$, and $\mathrm{O}$ atoms, respectively. For $k$-space integration and full convergence in the irreducible Brillouin zone (IBZ), $35 k$-points are used. Structural optimizations have been performed by a process of volume optimizations [43], see Fig. 2, 
which provides structural parameters such as lattice constant $a_{0}$, bulk modulus $B$ and its pressure derivative $B^{\prime}$, ground state volume $V$ and energy $E$ of unit cell (see Table I). The reduced crystal volume can be obtained from pressure $P$ volume variations in the Birch-Murnaghan equation of state given as [44]:

$$
P=\frac{B}{B^{\prime}}\left(\left(\frac{V_{0}}{V}\right)^{B^{\prime}}-1\right) .
$$

One can notice from Table I that lattice constant and bond length decrease with increase in pressure. The variation in bond lengths under normal pressure for $\mathrm{Pb}-\mathrm{Zr}$ (3.630 a.u.) and $\mathrm{Zr}-\mathrm{O}$ (2.095 a.u.) has decreased to (3.540 a.u.) and (2.044 a.u.), respectively, with the increase in applied pressure of $15 \mathrm{GPa}$. The aforementioned ground state parameters have been used to calculate optoelectronic properties of the compound.

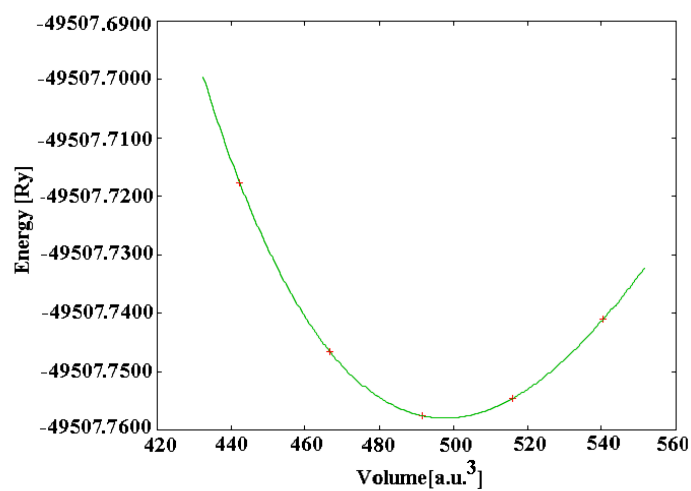

Fig. 2. Volume optimization curve of $\mathrm{PZO}$ at ambient pressure.

TABLE I

Optimized ground state structural properties of PZO at ambient pressure.

\begin{tabular}{|c|c|c|c|c|}
\hline \multirow{3}{*}{ Property } & \multicolumn{3}{|c|}{ Theory } & \multirow[t]{3}{*}{ Experiment } \\
\hline & \multirow{2}{*}{$\begin{array}{c}\text { this work } \\
\text { GGA }\end{array}$} & \multicolumn{2}{|c|}{ other works } & \\
\hline & & GGA & LDA & \\
\hline \multirow{3}{*}{$a_{0}[\AA]$} & \multirow{3}{*}{4.19} & 4.19 [41] & & \multirow{3}{*}{$4.16[18]$} \\
\hline & & 4.18 [40] & $\begin{array}{l}4.20[42] \\
411[25]\end{array}$ & \\
\hline & & 4.13 [42] & $4.11[20]$ & \\
\hline \multirow{2}{*}{$B$ [GPa $]$} & \multirow{2}{*}{158} & 138 [40] & $181[42]$ & \multirow{2}{*}{-} \\
\hline & & 168 [42] & & \\
\hline \multirow{2}{*}{$B^{\prime}$} & \multirow{2}{*}{4.13} & $6.4[40]$ & $4.6[42]$ & \multirow{2}{*}{-} \\
\hline & & $3.7[42]$ & & \\
\hline$V_{0}\left[(\text { a.u. })^{3}\right]$ & 496 & $492[40]$ & 499 [42] & - \\
\hline$E[\mathrm{Ry}]$ & -49507 & $\begin{array}{c}4.5[42] \\
-\end{array}$ & $\begin{array}{c}408[25] \\
-\end{array}$ & \\
\hline & & & & \\
\hline
\end{tabular}

\subsection{Electronic properties}

Figure 3 represents electronic band structures of PZO under different pressures. It has been observed that under ambient condition top of valence band (VB) and bottom of the conduction band $(\mathrm{CB})$ are located at $R$ and $\Gamma$ symmetry point, respectively. Hence, PZO regarded as

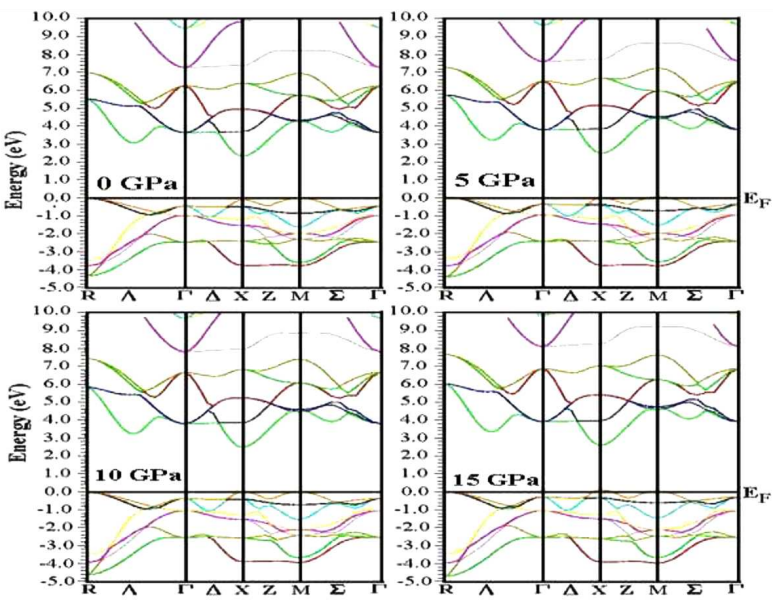

Fig. 3. Band structure of PZO for 0-15 GPa.

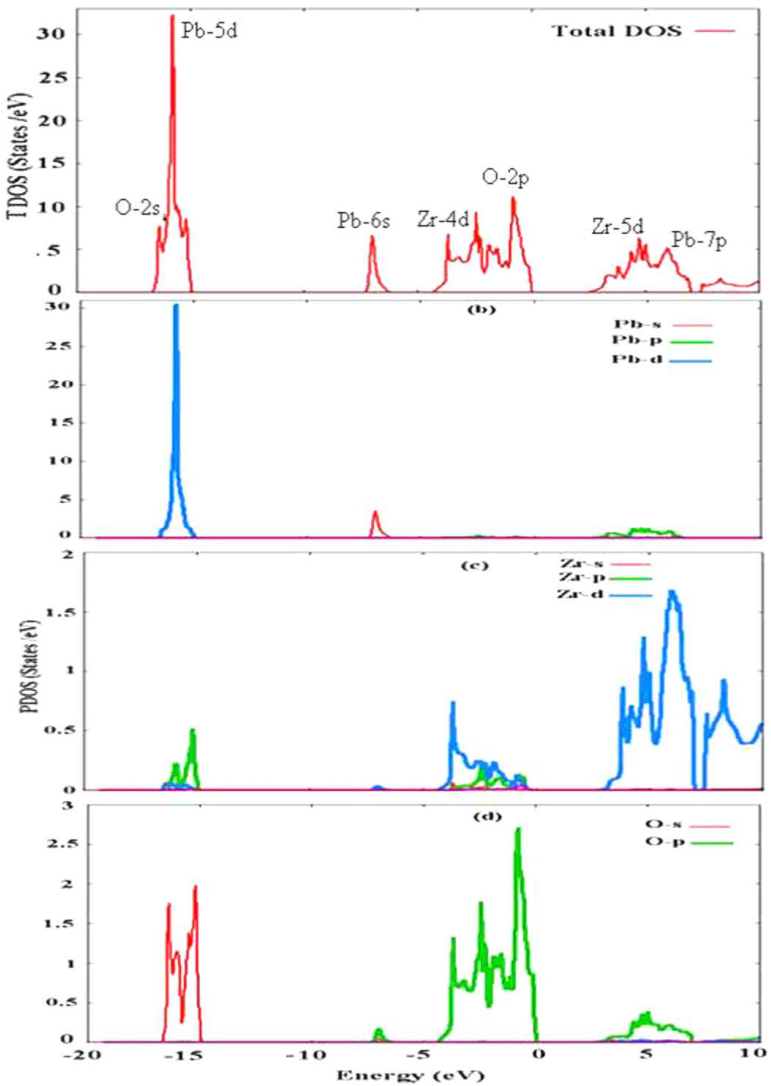

Fig. 4. Total and partial DOS of PZO under ambient pressure.

indirect band-gap semiconductor. Interestingly, while increasing the pressure at $15 \mathrm{GPa}$, shifting the top of VB at $\Gamma$ symmetry points towards the Fermi level was observed. Contrary to this, $M$ and $R$ symmetry points observed to move away from the Fermi level, this transformed the material into a direct band-gap semiconductor at $X$ symmetry point. The band-gap $E_{g}$ calculated from density of states (DOS) under ambient pressure and at $15 \mathrm{GPa}$ are $2.4 \mathrm{eV}$ and $2.6 \mathrm{eV}$, respectively, as shown in Fig. 4 and Fig. 5. It is well known that GGA functional un- 


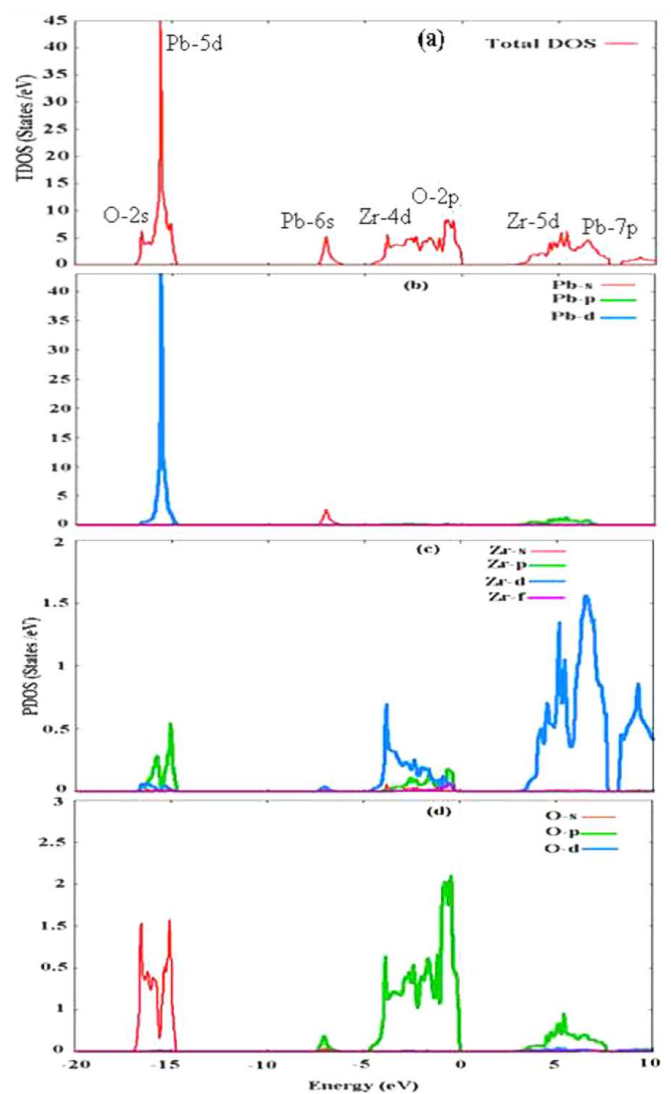

Fig. 5. Total and partial DOS of PZO under 15 GPa.

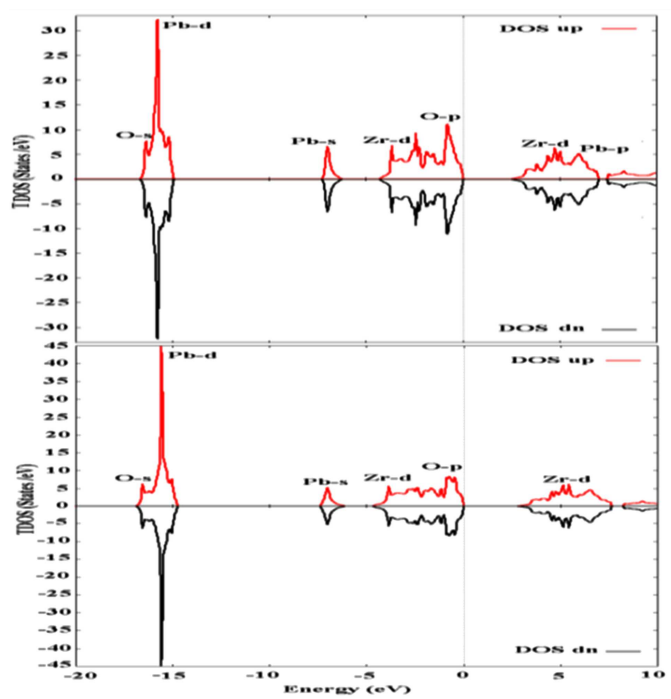

Fig. 6. Total DOS of PZO for spin up and spin down at normal pressure and $15 \mathrm{GPa}$.

derestimates in calculating the electronic band-gap, but the use of hybrid exchange-correlation functionals, such as B3LYP and B3PW, provides better results $[45,46]$.

DOS plots represents the inside picture of electronelectron correlation effects in a unit cell. This helps in understanding the complex bonding nature of the material. From Figs. 4, 5, it can be seen that DOS's can be

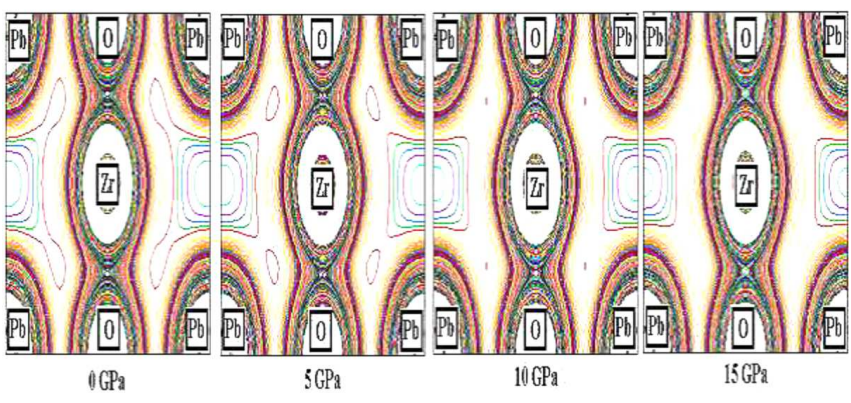

Fig. 7. Electron contour plots of PZO along (110) plane.

classified into four distinct regions. First region (core valence states) exhibits major contribution of $\mathrm{Pb} 5 d$ states and minor contribution from $\mathrm{O} 2 s$ ranges from $-16.8 \mathrm{eV}$ to $-15 \mathrm{eV}$ under $0 \mathrm{GPa}$ and ranges between $-17.2 \mathrm{eV}$ and $-14.8 \mathrm{eV}$ at $15 \mathrm{GPa}$. The second region contains $\mathrm{Pb} 6 s$ states ranges from $-7.2 \mathrm{eV}$ to $-6.2 \mathrm{eV}$ at $0 \mathrm{GPa}$ and from $-7.4 \mathrm{eV}$ to $-6.0 \mathrm{eV}$ at $15 \mathrm{GPa}$. In third region (near the Fermi level), major contribution comes from $\mathrm{O} 2 p$ states and minor contribution from $\mathrm{Zr} 4 d$ states falls in the same range $-4.6 \mathrm{eV}$ to $0.0 \mathrm{eV}$ under all pressures. 4th region is in $\mathrm{CB}$, here prominent major contribution is due to $\mathrm{Zr} 5 d$ states with a noticeable part of $\mathrm{Pb} 7 p$ states in range $2.4 \mathrm{eV}$ to $10 \mathrm{eV}$ at $0 \mathrm{GPa}$ and from $2.6 \mathrm{eV}$ to $10 \mathrm{eV}$ at $15 \mathrm{GPa}$.

We have also calculated the DOS for spin up and spin down electronic states to check the antiferromagnetic nature of PZO under ambient pressure and at $15 \mathrm{GPa}$ as shown in Fig. 6. We found that the DOS for spin up electronic states is invariant to DOS for spin down electronic states which shows non-magnetic nature of PZO under pressure range $0-15 \mathrm{GPa}$.

To visualize charge transfer and nature of bonding that correspond to the indirect nature of the PZO, electron charge density $2 \mathrm{D}$ contour plot in the plane direction (110) is shown in Fig. 7. It has been found that electronic charge transfer is in large amount, among $\mathrm{Pb}$ and $\mathrm{O}$ atoms. Due to this spherical contour extension around $\mathrm{Pb}$ and $\mathrm{O}$ depicts strong ionic character. Moreover, nonspherical electronic charge distribution around $\mathrm{Zr}$ and $\mathrm{O}$ shows that $\mathrm{Zr}-\mathrm{O}$ bond is strongly covalent in nature. The transformation of band-gap from indirect to direct can also be seen from Fig. 7. Here red contour lines show the interaction between electronic distribution among $\mathrm{Pb}$ and $\mathrm{Zr}$ atoms in the $\mathrm{BZ}$. These are $\mathrm{Pb} 6 s$ and $\mathrm{Zr} 4 d$ valence states. As the pressure increases, contours representing $\mathrm{Pb}$ and $\mathrm{Zr}$ valence states diminish and vanish completely at $15 \mathrm{GPa}$. This is due to the increase in ionic character and bond length between $\mathrm{Pb}$ and $\mathrm{Zr}$ atoms under pressure. Thus, we have now observed that the adjustment in the height of $R$ symmetry (as discussed before from Fig. 3) point near the Fermi level is due to these valence states $(\mathrm{Pb} 6 s$ and $\mathrm{Zr} 4 d)$.

\subsection{Optical properties}

Internal structure of materials can be discussed efficiently by considering its optical properties. A suitable 
material used in optoelectronic industry can be chosen by knowledge of its optical properties. Here we report optical properties for cubic phase PZO under pressure. Figures 8-16 shows optical parameters computed for cubic phase of PZO under pressure range 15-350 GPa calculated for energy range up to $45 \mathrm{eV}$. Figure 8 shows that inverse relation between optical band-gap and static dielectric constant validates the Penn model [47]. Furthermore, plasma energy determined from results obtained by using the Penn model are listed in Table II.

TABLE II

Optical properties of PZO under pressure 0-350 GPa.

\begin{tabular}{c|c|c|c|c}
\hline \hline$P[\mathrm{GPa}]$ & $\varepsilon_{1}(0)$ & $E_{g}[\mathrm{eV}]$ & $\omega_{p}$ & $n_{0}$ \\
\hline amb. & 4.526 & 2.39 & 1.3693 & 2.589 \\
15 & 4.452 & 2.597 & 1.2392 & 2.580 \\
100 & 4.496 & 2.489 & 1.0836 & 2.584 \\
250 & 4.555 & 2.095 & 1.4783 & 2.627 \\
350 & 4.570 & 1.574 & 1.8316 & 2.636
\end{tabular}

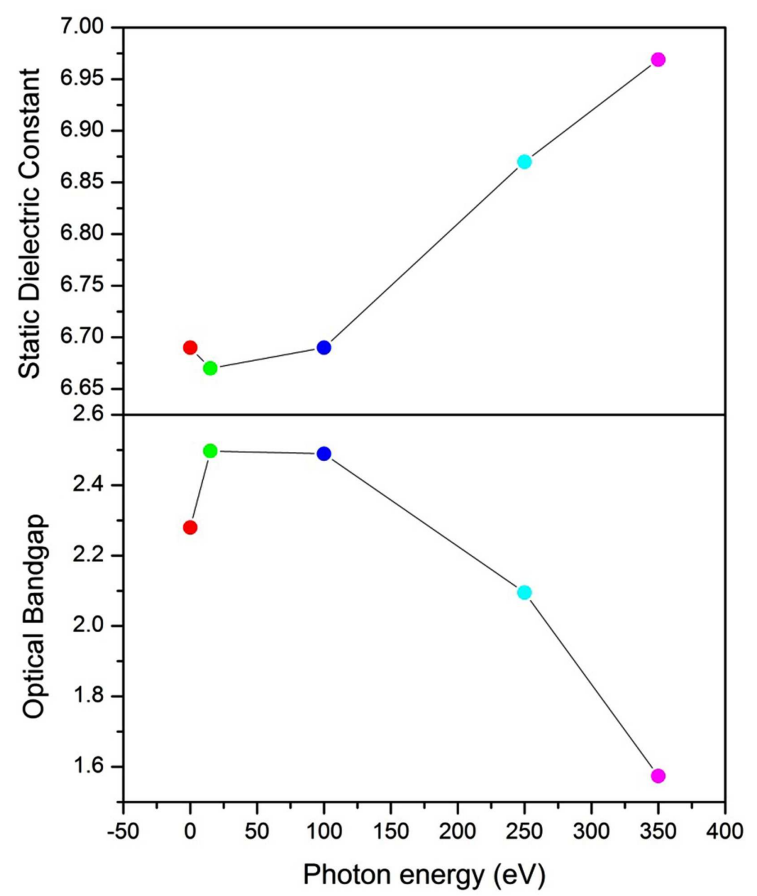

Fig. 8. Pressure variation calculation for static dielectric constant and optical band-gap for cubic phase $\mathrm{PbZrO}_{3}$.

The real and imaginary parts of dielectric function are shown in Fig. 9. The real part of dielectric function gives information about which extent a material may be polarized. It has been observed that the measured values of static dielectric constant depend upon the band-gap of the material. With increase in pressure from $15 \mathrm{GPa}$ to $350 \mathrm{GPa}$ peak value of static dielectric function increases from $3.775 \mathrm{eV}$ to $4.771 \mathrm{eV}$, while real dielectric function decreases and becomes zero at $6.825 \mathrm{eV}$ to $8.867 \mathrm{eV}$, respectively. On further increase in energy, it is observed that dielectric function becomes negative which shows

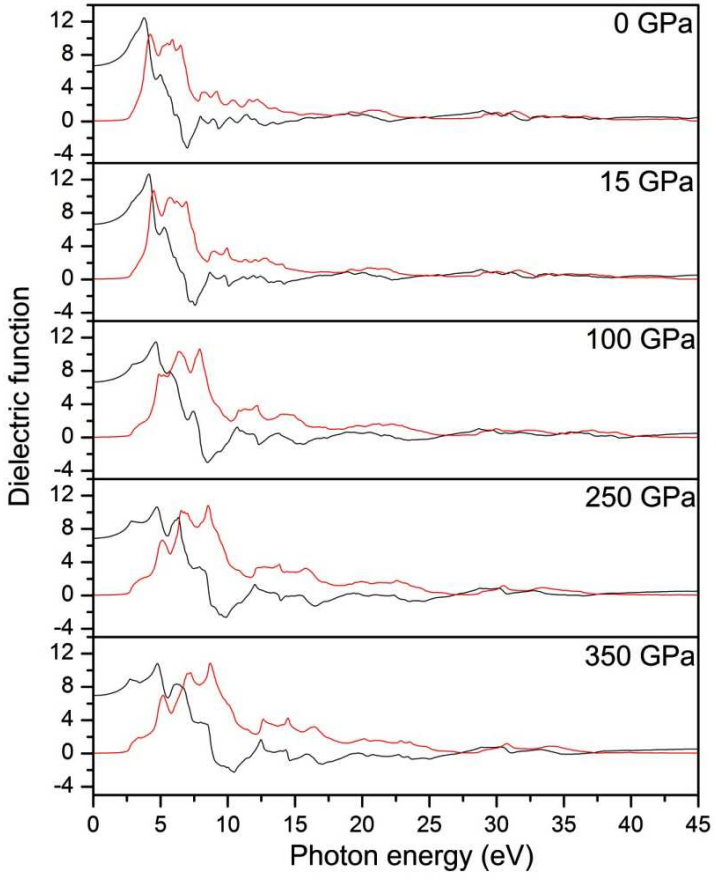

Fig. 9. Frequency dependent dielectric functions of cubic phase $\mathrm{PbZrO}_{3}$ at different values of pressure (real part: black lines, imaginary part: red lines).

that the medium reflects the entire incident light thus PZO behaves as metallic material.

The graph also shows a shift in hump toward the higher energies by increasing the value of pressure up to $350 \mathrm{GPa}$. Imaginary part of dielectric function contributes significant role in defining optical properties for any material. Large value corresponds to higher absorption of the medium. This suggests a deep impact on absorption. Figure 9 shows the behavior of dielectric function imaginary part versus energy. This shows that width of the absorption region of the material increases with increase in pressure. Maximum absorption takes place in energy range of $2.213 \mathrm{eV}$ to $37.030 \mathrm{eV}$ at $350 \mathrm{GPa}$ and minimum absorption at $15 \mathrm{GPa}$. These are attributed to the direct band-gap transition at high symmetry points among the maxima of valence band and minima of the conduction band. These regions consist of different peaks which exist because of inter-band transition among the valence and conduction band.

In addition, to study the surface behavior of material, reflectivity is determined by considering the ratio of incident to reflected power. Figure 10 represents zero frequency limits of reflectivity; it exhibits an elevation with the rise in pressure and reaches optimum peak value at $350 \mathrm{GPa}$ of $25.297 \mathrm{eV}$. The appearance of these peaks is attributed to the inter-band transition among valence and conduction bands. In contrast, energy ranging from $25 \mathrm{eV}$ to $30 \mathrm{eV}$ exhibits the minimum value of reflectivity as a consequence of collective plasma resonance. Moreover, the plasma resonance can be determined by imaginary part of dielectric function [48]. Peak in reflectivity 


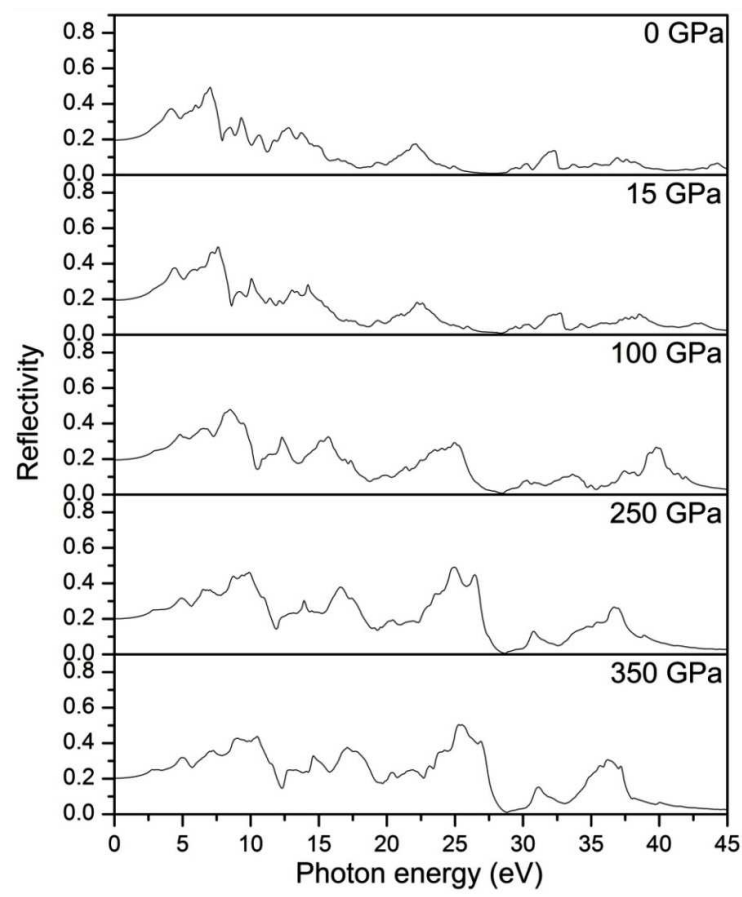

Fig. 10. Frequency dependent reflectivity of cubic phase $\mathrm{PbZrO}_{3}$ at different values of pressure.

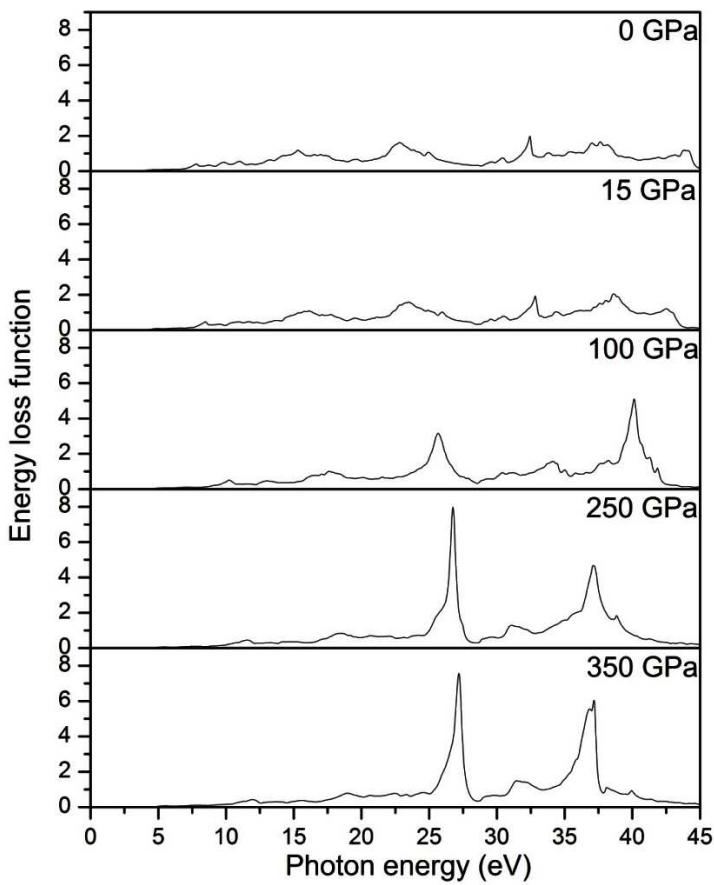

Fig. 11. Frequency dependent energy loss function of cubic phase $\mathrm{PbZrO}_{3}$ at different values of pressure.

shifted towards higher energy as pressure is raised consistent with the dielectric function imaginary part $[49,50]$.

Figure 11 shows energy loss function under different pressures. The prominent peaks in the graph give a detail interrogation about properties associated with plasma resonance or plasma frequency. No energy loss was ob- served for the photons owing energy less than $7.182 \mathrm{eV}$ to $9.248 \mathrm{eV}$ for $15 \mathrm{GPa}$ to $350 \mathrm{GPa}$. For further increase in photon energy, energy loss starts to increase and reaches the maximum peak value in energy range $38.604 \mathrm{eV}$ to $27.179 \mathrm{eV}$ for $15 \mathrm{GPa}$ to $350 \mathrm{GPa}$. It can be seen that highest peak shifts toward higher value of energy with the increase in pressure up to $100 \mathrm{GPa}$, with the further increase in pressure, the peak shifts toward the lower value of energy as can be seen from the figure at $250 \mathrm{GPa}$ and then again on further increase in pressure, it goes toward higher energy value at $350 \mathrm{GPa}$.

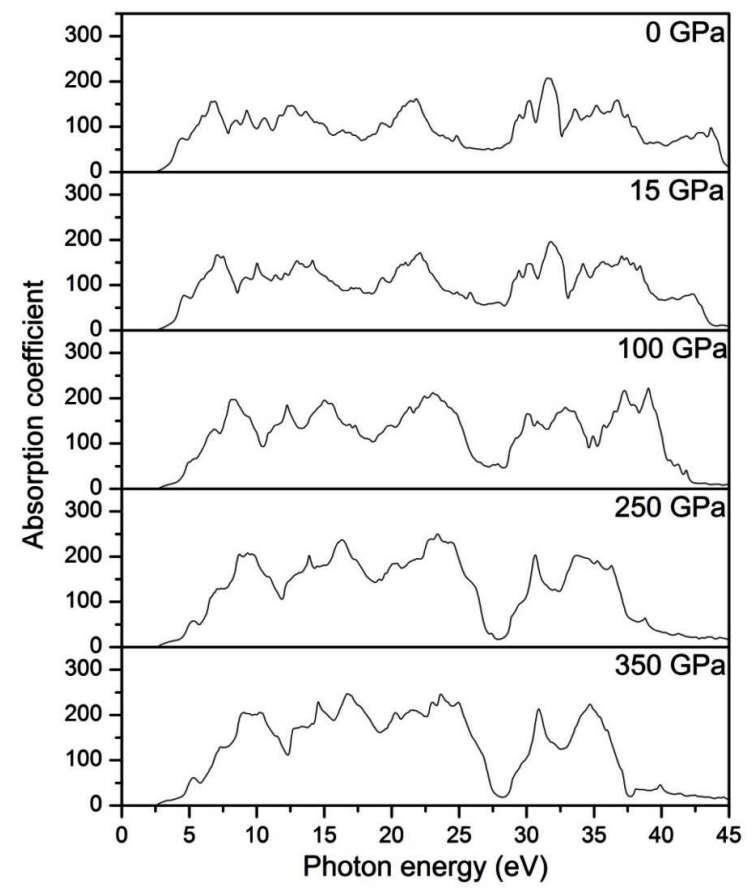

Fig. 12. Frequency dependent absorption coefficient of cubic phase $\mathrm{PbZrO}_{3}$ at different values of pressure.

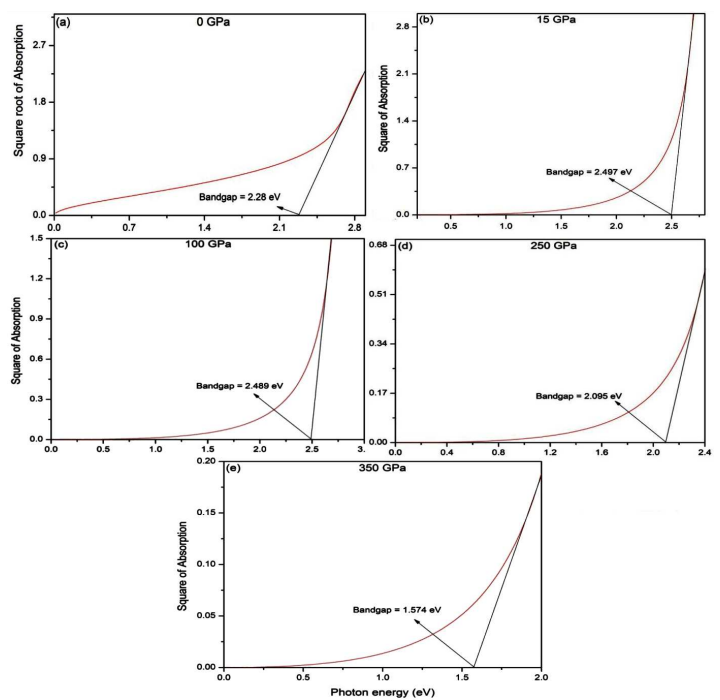

Fig. 13. Optical band gap of cubic phase $\mathrm{PbZrO}_{3}$ at different values of pressure. 


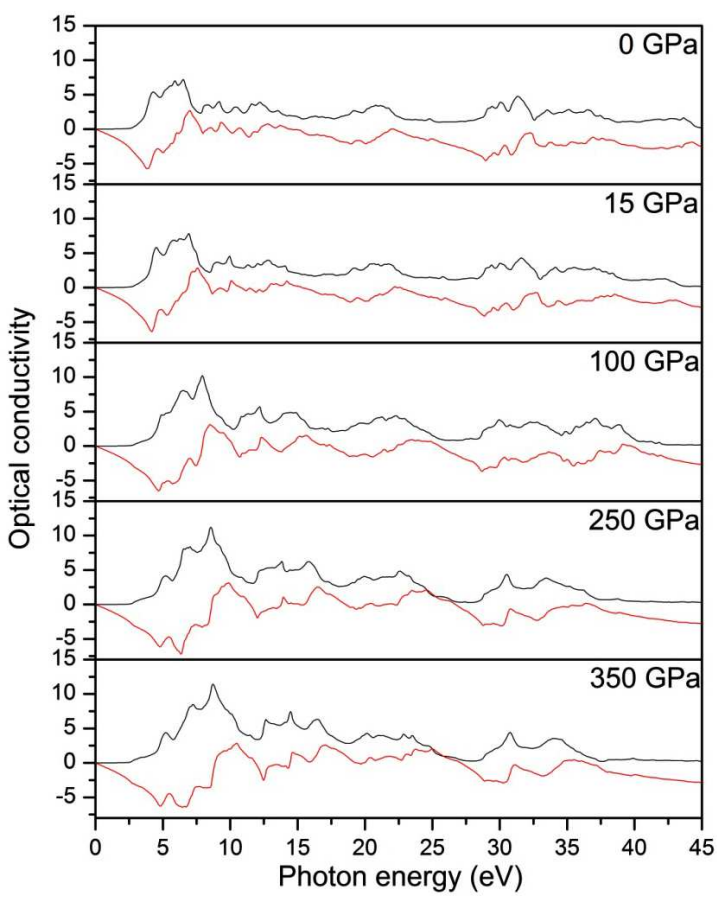

Fig. 14. Frequency dependent optical conductivity of cubic phase $\mathrm{PbZrO}_{3}$ at different values of pressure (real part: black lines, imaginary part: red lines).

The highest peak $(8.0 \mathrm{eV})$ occurs at $26.761 \mathrm{eV}$ for $250 \mathrm{GPa}$. The observed peak in the energy loss function is associated with the plasma frequency and can be treated as an interface between metallic and dielectric behavior. Absorption coefficient is another important factor which represents the decay of light intensity per unit distance through medium. Figure 12 represent absorption coefficient as a function of frequency for cubic phase of PZO at different pressure values. The variation in absorption is described in terms of energy range from 0 up to $45 \mathrm{eV}$. The highest peaks occur at $31.779,39.022$, 23.391, $16.713 \mathrm{eV}$ for pressure 15, 100, 250, and $350 \mathrm{GPa}$, respectively. Therefore, we observed that by increasing pressure, the absorption peak shifts toward higher value of energy up to $100 \mathrm{GPa}$, with further increasing pressure, it goes toward lower value of energy as shown in the figure. It has also been illustrated that no absorption is observed for photon energy less than 2.877, 2.951, $3.025,2.877,2.804 \mathrm{eV}$ for $0,15,100,250$, and $350 \mathrm{GPa}$ pressures, respectively. However, with photon energy greater than these values, absorption coefficient starts to increase, which is associated with the direct band-gap values $2.28,2.497,2.489,2.095,1.574 \mathrm{eV}$ calculated for cubic phase PZO at different high pressures, respectively.

Figure 13 shows optical band-gap values determined by knowing the direct and indirect behavior of the cubic phase PZO at different values of pressure by using theoretical approach of square of absorption. The real and imaginary part of optical conductivity is also studied for cubic phase PZO as shown in Fig. 14. We can see that real part of conductivity is zero for energy values: 2.656, 2.693, 2.791, 2.693, 2.631 eV at pressures 0, 15, 100, 250,

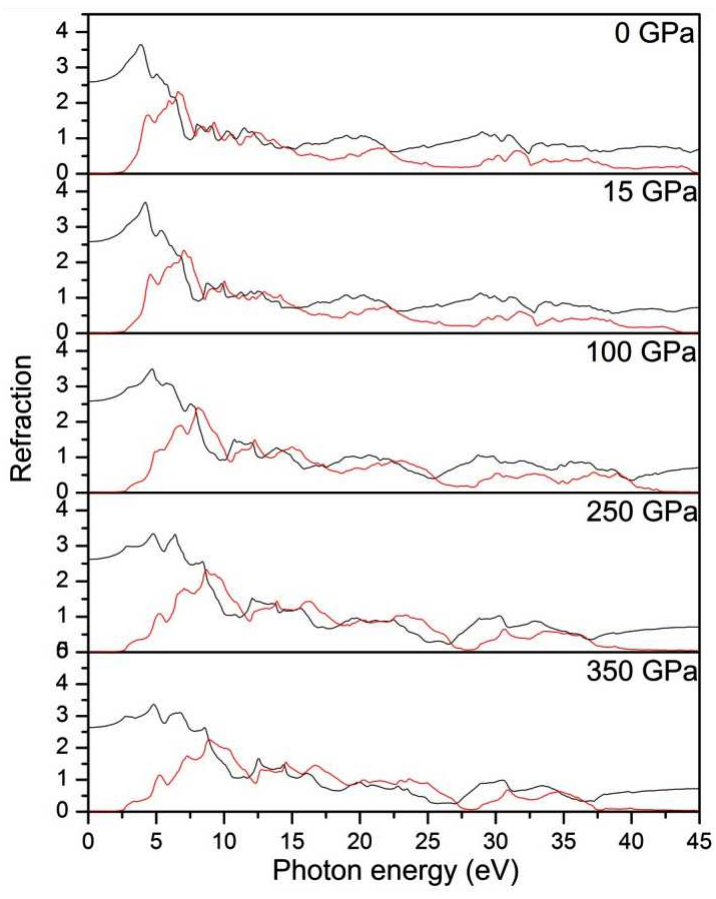

Fig. 15. Frequency dependent refractive indices of cubic phase $\mathrm{PbZrO}_{3}$ at different values of pressure (refractive index: black lines, extinction coefficient: red lines).

and $350 \mathrm{GPa}$. This is in good agreement with the bandgap of the material at different pressure values. When the photon energy exceeds these values, the real part of conductivity starts to rise and exhibits the maximum peaks at $6.505,6.924,7.920,8.572,8.707 \mathrm{eV}$ for pressure of 0 , $15,100,250$, and $350 \mathrm{GPa}$, respectively. We can see that as the pressure is increased to higher values, the peak shift toward higher value of energy up to $350 \mathrm{GPa}$. After getting highest peak, it starts to decrease with the further increase in photon energy.

Figure 15 shows spectrum for refractive index and extinction coefficient versus energy. Figure also shows that the spectrum of dielectric constant nearly follows pattern as in Ref. [51].

It can be seen from the figure that static refractive index $n_{0}$ values are $2.589,2.580,2.584,2.627,2.636$ for cubic phase PZO at $0,15,100,250$, and $350 \mathrm{GPa}$ values of pressure which is in accordance with the static dielectric function. We can see that the peak in the refractive index shifts toward higher value of energy as the pressure exceeds from 0 to $350 \mathrm{GPa}$. In the middle of the graph, we can see different humps which vanish at higher values of photon energy. Consequently, it is attributed to the fact that beyond a certain value of energy, the transparent nature of material will no longer be maintained, and high energy photons are ready to be absorbed. At certain value of energy, the refractive index exhibit values lower than unity as shown in Fig. 15. Refractive index with value less than unity illustrates that group velocity possessed by incident radiation turn out to be greater than speed of light. 


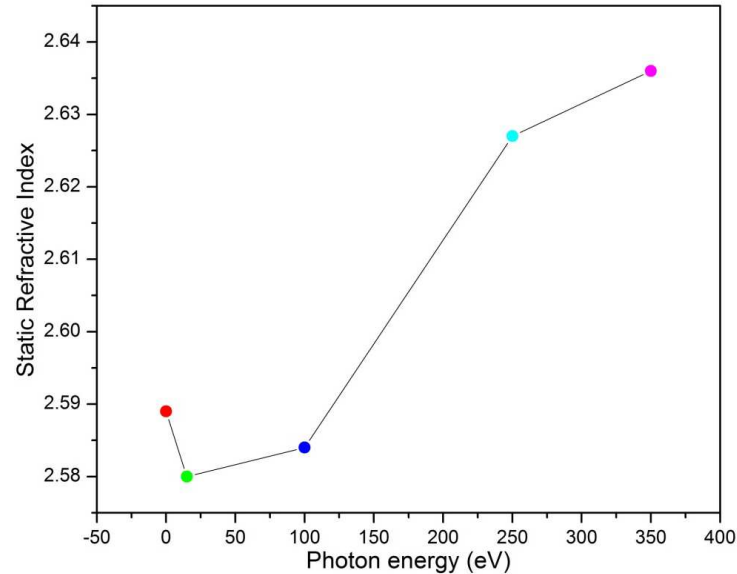

Fig. 16. Pressure variation of static refractive index for cubic phase $\mathrm{PbZrO}_{3}$.

This shows that group velocity exhibits a shift towards negative domain making the nature of medium non-linear. Hence, we can conclude that the material transforms to superluminal medium for high energy photons $[52,53]$. The extinction coefficient is also shown in Fig. 15. The extinction coefficient gives idea about the absorption of light, and at the same time, absorption characteristics at the band extremes. The peaks exist in refractive index and in extinction coefficient are possibly due to transition of electrons among valence and conduction bands. The extinction coefficient response is closely related to imaginary part of dielectric function at different values of pressure [51]. Figure 16 shows the response of static refractive index under different pressure values.

\section{Conclusion}

PZO in cubic phase has been explored in terms of its properties including structure and optoelectronics under pressure range 0 to $350 \mathrm{GPa}$. The outcome of this study at ambient pressure agreed well with the literature. It has been observed that with increase in hydrostatic pressure lattice constant and bond length have been observed to decrease. While at $15 \mathrm{GPa}$, the adjustment of electronic states $\mathrm{Pb} 6 s$ and $\mathrm{Zr} 4 d$ due to strong ionic character, transformed the material into a direct band-gap semiconductor. With further increase in pressure up to $350 \mathrm{GPa}$ static dielectric constant, plasma frequency, static refractive indices increases with the pressure linearly. However, the decrease in optical band-gap with the increase in pressure showed consistency with the Penn model. Throughout positive value of refractive index portrays that PZO had not changed into negative index meta-material under pressure. Plasma frequency has also been observed to increase with pressure, this exhibit non-stability of the material under extreme pressure. Our reported results would be very useful in understanding and developing useful optoelectronic devices based on PZO to work under extreme conditions.

\section{Acknowledgments}

Asif Mahmood would like to extend his sincere appreciations to the Deanship of Scientific Research at King Saud University for funding this Prolific Research Group (PRG-1436-26).

\section{References}

[1] J.F. Scott, Science 315, 954 (2007).

[2] E. Cross, Nature 432, 24 (2004).

[3] T. Schneider, D. Leduc, J. Cardin, C. Lupi, N. Barreau, H. Gundel, Opt. Mater. 29, 1871 (2007).

[4] R. Watton, Ferroelectrics 91, 87 (1989).

[5] R.W. Whatmore, Ferroelectrics 118, 241 (1991).

[6] M.E. Lines, A.M. Glass, Principles and Applications of Ferroelectrics and Related Materials, Oxford University Press, Oxford 1977.

[7] C. Noguera, Physics and Chemistry at Oxide Surfaces, Cambridge University Press, Cambridge 1996.

[8] K. Uchino, Ferroelectric Devices, 2nd ed., CRC Press, 2009.

[9] Y. Xu, Ferroelectric Materials and Their Applications, North-Holland Elsevier Sci., Amsterdam 1991.

[10] F. Jona, G. Shirane, F. Mazzi, R. Pepinsky, Phys. Rev. 105, 849 (1957).

[11] R.W. Whatmore, A.M. Glazer, J. Phys. C Solid State Phys. 12, 1505 (1979).

[12] E. Sawaguchi, J. Phys. Soc. Jpn. 8, 615 (1953).

[13] H. Fujishita, Y. Shiozaki, N. Achiwa, E. Sawaguchi, J. Phys. Soc. Jpn. 51, 3583 (1982).

[14] M. Tanaka, R. Saito, K. Tsuzuki, J. Phys. Soc. Jpn 51, 2635 (1982).

[15] H. Fujishita, S. Hoshino, J. Phys. Soc. Jpn. 53, 226 (1984).

[16] H. Fujishita, S. Katano, Ferroelectrics 237, 209 (2000).

[17] H. Fujishita, S. Tanaka, Ferroelectrics 258, 37 (2001).

[18] H. Fujishita, Y. Ishikawa, S. Tanaka, A. Ogawaguchi, S. Katano, J. Phys. Soc. Jpn. 72, 1426 (2003).

[19] D.J. Singh, Phys. Rev. B 52, 12559 (1995).

[20] M.D. Johannes, D.J. Singh, Phys. Rev. B 71, 212101 (2005).

[21] E. Sawaguchi, Ferroelectrics 266, 5 (2002).

[22] H. Fujishita, Ferroelectrics 266, 27 (2002).

[23] S. Teslic, T. Egami, Acta Crystallogr. B Struct. Sci. 54, 750 (1998).

[24] D. Vanderbilt, Curr. Opinion Solid State Mater. Sci. 2, 701 (1997)

[25] Y.X. Wang, M. Arai, T. Sasaki, C.L. Wang, W.L. Zhong, Surf. Sci. 585, 75 (2005).

[26] M.D. Johannes, D.J. Singh, Phys. Rev. B 71, 212101 (2005).

[27] P.S.H. Ghosez, X. Gonze, J.P. Michenaud, Ferroelectrics 206, 205 (1998).

[28] D.J. Singh, Phys. Rev. B 52, 12559 (1995). 
[29] D.J. Singh, L. Nordstrom, Planewaves, Pseudopotentials, and the LAPW Method, Springer Sci. \& Business Media, 2006.

[30] J.P. Perdew, K. Burke, M. Ernzerhof, Phys. Rev. Lett. 77, 3865 (1996).

[31] K. Schwarz, P. Blaha, Comput. Mater. Sci. 28, 259 (2003).

[32] S. Cottenier, Density Functional Theory and the Family of (L)APW-Methods: A Step-by-Step Introduction, Vol. 4, Instituut voor Kern-en Stralingsfysica, KU Leuven, Belgium 2002, p. 41.

[33] H. Ehrenreich, M.H. Cohen, Phys. Rev. 115, 786 (1959).

[34] D. Groh, R. Pandey, M.B. Sahariah, E. Amzallag, I. Baraille, M. Rerat, J. Phys. Chem. Solids 70, 789 (2009).

[35] S. Tariq, A. Afaq, S. Saad, S. Tariq, AIP Adv. 5 077111 (2015).

[36] G. Nazir, A. Ahmad, M.F. Khan, S. Tariq, Computational Condensed Matter 4, 32 (2015).

[37] S. Nadeem, S. Tariq, M.I. Jamil, E. Ahmad, S.S. Gilani, K.S. Munawar, J. Theor. Comput. Chem. 15, 1650044 (2016); S. Tariq, M.I. Jamil, A. Sharif, S.M. Ramay, H. Ahmad, N. ul Qamar, B. Tahir, Appl. Phys. A. 124, (2018).

[38] Y. Syono, M.H. Manghnani, High-Pressure Research: Application to Earth and Planetary Sciences, Vol. 3, American Geophysical Union, 1992.

[39] Y. Kobayashi, S. Endo, L.C. Ming, K. Deguchi, T. Ashida, H. Fujishita, J. Phys. Chem. Solids 60 57 (1999).
[40] J. Baedi, S.M. Hosseini, A. Kompany, E.A. Kakhki, Phys. Status Solidi B 245, 2572 (2008).

[41] X.G. Tang, H.R. Zeng, A.L. Ding, P.S. Qiu, W.G. Luo, H. Li, D. Mo, Solid State Commun. 116, 507 (2000).

[42] N. Pandech, K. Sarasamak, S. Limpijumnong, Ceram. Int. 39, S277 (2013).

[43] G. Murtaza, I. Ahmad, B. Amin, A. Afaq, M. Maqbool, J. Maqssod, M. Zahid, Opt. Mater. 33, 553 (2011).

[44] F. Birch, Phys. Rev. 71, 809 (1947).

[45] R.I. Eglitis, M. Rohlfing, J. Phys. Condens. Matter 22, 415901 (2010).

[46] R.I. Eglitis, Appl. Surf. Sci. 358, 556 (2015).

[47] D.R. Penn, Phys. Rev. 128, 2093 (1962).

[48] A.H. Reshak, Z. Charifi, H. Baaziz, Europ. Phys. J. B Condens. Matter Complex Syst. 60, 463 (2007).

[49] B. Amin, I. Ahmad, M. Maqbool, J. Lightwave Technol. 28, 223 (2010).

[50] R. Khenata, M. Sahnoun, H. Baltache, M. Rerat, A.H. Rashek, N. Illes, B. Bouhafs, Solid State Commun. 136, 120 (2005).

[51] M. Fox, Optical Properties of Solids, 2nd ed., Oxford University Press, Oxford 2010.

[52] L.J. Wang, A. Kuzmich, A. Dogariu, Nature 411, 974 (2001).

[53] D. Mugnai, A. Ranfagni, R. Ruggeri, Phys. Rev. Lett. 84, 4830 (2000). 